\title{
Article \\ Wave Steepness from Satellite Altimetry for Wave Dynamics and Climate Studies
}

\author{
Sergei I. Badulin ${ }^{1,2, t, t *(\mathbb{C})}$, Vika G. Grigorieva ${ }^{1, t, \ddagger}(\mathbb{D})$, Alexander V. Gavrikov $1, t, \ddagger *(\mathbb{D})$, Vladimir V.

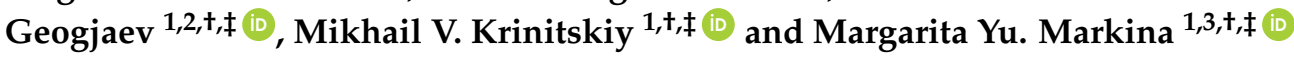 \\ 1 Shirshov Institute of Oceanology, Russian Academy of Sciences; \\ 2 Laboratory of Nonlinear Wave Processes, Novosibirsk State University, Russia; \\ 3 Lomonosov Moscow State University, Russia \\ * Correspondence: badulin.si@ocean.ru; Tel.: +7-916-379-6322 \\ + Current address: 36, Nahimovskiy pr., 117997, Moscow, Russia \\ $\ddagger$ These authors contributed equally to this work.
}

\begin{abstract}
Wave steepness is presented as an extension and a valuable add-on to the conventional set of sea state parameters retrieved from satellite altimetry data. Following physical model based on recent advances of weak turbulence theory wave steepness is estimated from directly measured spatial gradient of wave height. In this way the method works with altimetry trajectories rather than with point-wise data. Moreover, in contrast to widely used parametric models this approach provides us with instantaneous values of wave steepness and period. Relevance of single-track estimates of wave steepness (period) is shown for wave climate studies and confirmed by a simple probabilistic model. The approach is verified via comparison against buoy and satellite data including crossover points for standard 1 second data of Ku-band altimeters. High quality of the physical model and robustness of the parametric ones are examined in terms of global wave statistics. Prospects and relevance of both approaches in the ocean wave climate studies are discussed.
\end{abstract}

Keywords: wave turbulence; satellite altimetry; wave steepness; parametric and physical models of wave period.

\section{Introduction}

Sea waves is a complex physical phenomenon that affects (and is affected by) a number of processes in a wide range of spatial and temporal scales: from small-scale turbulence to large-scale ocean circulation. Wind waves which are driven mostly by local winds contribute into the ocean dynamics at relatively short scales of stormy regions while ocean swells transfer energy over thousands of miles (e.g. from the Roaring Forties to the Northern Pacific) and thus can be regarded as a large-scale process.

Conventional in situ measurements are not always relevant to the physical phenomenon of sea waves being essentially local, scarce in time and space, and not accurate enough. In most cases this data provides two key wave parameters (sometimes, along with wind observations) for prediction and diagnosis of wind-wave coupling: significant wave height and wave period (mean, peak, zero-crossing etc.).

Remote sensing methods do not measure sea waves directly. Two general approaches are used to convert the measurable quantities into characteristics of sea state. Firstly, the conventional procedures of calibration and validation provide a basis for empirical models of sea state from remote sensing data. An alternative way relies upon essential physical links between the measured parameters and those being estimated. The latter approach leads to the development of physical models of sea state [1]. These two approaches are widely used in satellite altimetry both individually and more often in a combination.

The model of electromagnetic scattering by a gaussian random sea surface provides a reference shape of the altimeter echo [2]. As the very first approximation the sea state parameters can be retrieved 
9

through fitting the measured echo to this shape [e.g. 3]. The tangent of the leading edge of the echo is associated with sea surface roughness and can be quantified by significant wave height $H_{s}$. The normalized radar cross-section $\sigma_{0}$ is another parameter measured directly which is affected heavily by the sea surface roughness, sea spray from wave breaking, sea surface pollution etc.. The latter makes $H_{s}$ and $\sigma_{0}$ essentially correlated and constrains the application of the pair $\left(H_{s}, \sigma_{0}\right)$ for empirical models of wind speed and wave period [see discussion in 1]. This correlation can be regarded as a spurious one which effect is difficult to be controlled and evaluated.

In a way the wave height can be regarded as a cleaner parameter in comparison with $\sigma_{0}$. Wave height $H_{s}$ and its spatial gradient $\nabla H_{s}$ were suggested by the physical model of Badulin [1, hereinafter B14] as a new combination of measured parameters that leads to the following expression for the spectral peak period $T_{p}[1$, eq.6]

$$
T_{p}=2^{1 / 5} \pi \alpha_{s s}^{-3 / 10} \sqrt{H_{s} / g}\left|\nabla H_{s}\right|^{-1 / 10} .
$$

The expression (1) does not refer to any empirical quantities but to the gravity acceleration $g$ and the dimensionless physical parameter $\alpha_{S S}$ which is a direct equivalent of the fundamental Kolmogorov-Zakharov constant in the theory of wave turbulence [4-6]. Technically two crossover measurements of satellite altimeters are required for obtaining the vector module $\left|\nabla H_{s}\right|$ in (1) and thus for an accurate estimate of wave period. Fortunately, low exponent $1 / 10$ mitigates this requirement making the along-track measurements by a single altimeter acceptable in many cases of interest.

Being rewritten for $\mu$ the model B14 provides a remarkably simple dependence on spatial gradient $\nabla H_{s}$

$$
\mu=\frac{\alpha_{s s}^{3 / 5}}{2^{2 / 5}}\left|\nabla H_{S}\right|^{1 / 5} \approx 0.596\left|\nabla H_{s}\right|^{1 / 5}
$$

Here and after we refer to the steepness definition based on integral parameters of sea state, namely peak period $T_{p}$ and significant wave height $H_{s}$

$$
\mu=\frac{\pi^{2} H_{s}}{g T_{p}^{2}}
$$

The measured $H_{s}$ and empirically estimated $T_{p}$ in (3) can be essentially correlated while the alternative estimate (2) of wave steepness is free of any spurious correlations. The accuracy of the gradient $\nabla H_{S}$ measurements by altimetry is not an unsolvable issue for the modern technologies. Thus, the passage to the tandem $\left(H_{s}, \nabla H_{s}\right)$ is seen as a conceptual step that introduces 'a theoretical' wave steepness (2) into the conventional set of sea state parameters. This turn has not been presented before in B14. Also the method itself has not been substantiated properly by comparison with in situ data. The paper is aimed to fill in this gap.

In sect. 2 we discuss the B14 model in terms of the analogy with the model of geostrophic currents in order to specify advantages and disadvantages of the novel approach.

In sect.3 the new method is validated on Globwave satellite altimetry data (http://globwave.ifremer.fr/). We demonstrate the correspondence of the results to the today's understanding of sea wave climatology [7] as well as 'device-independent features' of the new approach. Global maps of wave steepness are presented and analyzed. Possible applications of the new approach in reconstructions of wind wave climate are discussed. The paper is summarised by the discussion of the prospects of the new method.

\section{Two models - one principle for satellite altimetry}

In this section we discuss two physical models of satellite altimetry both based on along-track gradients of parameters measured by altimeter. 

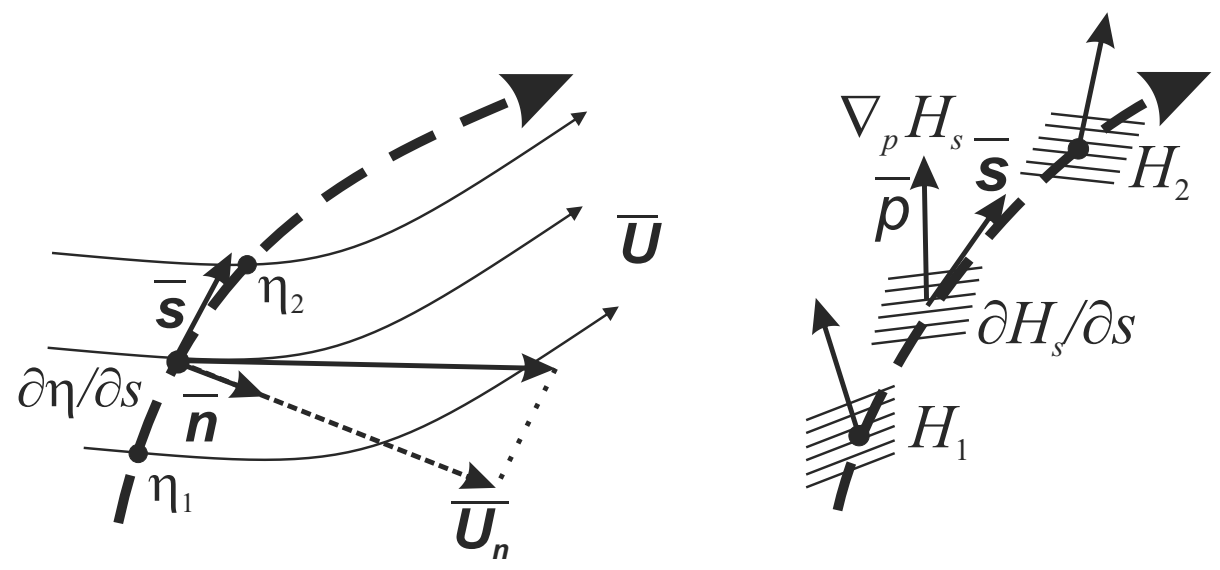

Figure 1. Left - setup of measurements of large-scale currents by satellite altimeter; right - setup of estimate of wave steepness from two consecutive along-track measurements of significant wave height by satellite altimeter.

\subsection{Gradients of sea surface height for monitoring geostrophic currents}

The well-known method of dynamical heights for large-scale currents (fig.1, left) considers the geostrophic balance of the gravity and the Coriolis forces

$$
2 \Omega \mathbf{U n} \sin \phi=-g \frac{\partial \eta}{\partial \mathbf{s}} \mathbf{s}
$$

where $2 \Omega=f$ is the Coriolis parameter and $\phi$ is the latitude of the site of measurements. The general expression (4) says that the along-track derivative of the hydrodynamic pressure (the tangent unit vector s) is balanced by the horizontal component of the current velocity Un which is normal to the track (the normal vector $\mathbf{n}$ in eq.4). A single satellite track provides the only along-track component of the pressure field gradient (more specifically, sea surface elevation relatively to equipotential level) and hence a single component of the velocity $U_{n}=\mathbf{U n}$ in the cross-track direction. The full vector $\mathbf{U}$ can be derived from two intersecting tracks. Obviously, time interval between these two consecutive along-track measurements should be small enough.

Linearity of the basic relation (4) between measured (surface elevation $\eta$ ) and estimated (current velocity $\mathbf{U}$ ) values can be considered as a fortunate coincidence. In this case, the relationship between the errors in sea level measurements and the accuracy of geostrophic current estimates is very simple. Theoretically, the accuracy of the estimates of geostrophic currents is limited by wavelength of the sounding pulse that is approximately $2.2 \mathrm{~cm}$ for the Ku-band and $0.8 \mathrm{~cm}$ for the recently launched Ka-band altimeter SARAL/AltiKa. An additional advantage of this method comes from spatial scales of the phenomenon under study (large-scale currents) being large enough to reduce noise of altimeter data when averaged.

\subsection{Gradients of sea surface roughness for wave studies}

Sea wave measurements require significantly shorter scales of averaging (both in time and space). The corresponding data is noisier and thus incomparably less accurate. However the accuracy of 
significant wave height measured by modern altimeters is better than $20 \mathrm{~cm}$ which is close to the accuracy of ocean buoys or better than one of visual observations.

Fig.1 illustrates a similarity of physical principles of altimetry measurements of large-scale currents (previous section and eq.4) and wave steepness with the model B14 (eq.2). Measurements in two consecutive points give an estimate of the corresponding directional derivative. For the geostrophic current this derivative is converted to the cross-track component of current velocity via linear relationship (4). A similar but heavily nonlinear conversion (2) of an unspecified projection of a spatial gradient gives a lower-bound estimate of wave steepness $\mu$. The full vector $\nabla H_{s}$ and hence a 'full' wave steepness $\mu$ can be obtained in crossover points of two altimeters. These cases are really rear because of the spatial and temporal requirements for mismatch in sea waves. Globwave database accepts mismatch of 30 minutes in time and 50 kilometers in space for the crossover points (http://globwave.ifremer.fr/products/globwavesatellite-data/satellite-crossovers) while spatial and temporal scales of wave field variability can be essentially shorter. The issue of physical scales becomes of key importance when considering the relevance of the method.

\subsection{Physical constraints for altimetry measurements of sea state}

A certain hierarchy of physical scales is implied for the model B14 validity. The realization of the method should respect this ranking in hardware parameters, options of measurements, and data processing.

A characteristic wavelength $\lambda$ (wave period $T_{p}$ ) is the shortest physical scale within the weakly nonlinear statistical description of water waves where the physical scale of wave-wave interactions obeys the well-known relationship [8]

$$
L_{n l} \sim \mu^{-4} \lambda .
$$

Even rather high steepness $\mu \simeq 0.1$ gives a large factor $10^{4}$ in (5) that induces a long-lasted discussion on the relevance of statistical approach for wind-driven seas. In fact, the accurate estimate of the proportionality coefficient in (5) reduces the scale of wave-wave interactions by two orders of magnitude [see eqs.17,18 in 9] that lets $L_{n l}$ to be a few kilometers for typical sea state.

Similar estimates of scales of wind wave growth from existing parametric models [e.g. 10] leads to the following relationship

$$
L_{\text {wind }} \sim \frac{\rho_{w}}{\rho_{a}} \lambda^{1+n}
$$

where $\rho_{w}$ and $\rho_{a}$ are water and air densities and $1 \leq n \leq 2$. The quantitative comparison of $L_{n l}$ and $L_{\text {wind }}$ leads to a key realization: wind wave growth occurs at scales which are, typically, longer (or even much longer) than scales of nonlinear relaxation of water waves [see fig.3 in 9]. It justifies validity of the asymptotic theory of wave growth $[5,11]$ when

$$
L_{n l} \leq L_{\text {wind }} .
$$

The satellite altimetry assumes a sufficiently large footprint $L_{a}$ of few kilometers. To be consistent with the statistical description of sea wave field $L_{a}$ should be close or larger than the scale of the wave field relaxation associated with the fastest physical mechanism of wave-wave interactions, i.e.

$$
L_{a} \geq L_{n l} .
$$

A new physical scale $\Delta L$ appears when computing spatial gradient of wave height $H_{s}$ between two consecutive footprints. This scale should be close to or larger than the footprint size $L_{a}$ to ensure independence of two consecutive measurements. On the other hand, $\Delta L$ should be less (much less) 
than the scale of wave field variations associated with wind input and dissipation. Finally, we come to the following sequence of physical scales (cf. B14, eq.11)

$$
L_{n l} \leq L_{a} \leq \Delta L \ll L_{\text {wind }}
$$

In practice, the formal requirement 'much less' ( $\ll$ ) at the very end of the sequence (8) can be replaced by a more flexible condition of simple inequality but the gap between two key physical scales of sea state, $L_{n l}$ and $L_{\text {wind }}$, should exist and scales of altimeter measurements, $L_{a}$ and $\Delta L$, should fall into this gap for the method to work. The critical point of conditions (8) is an essential dependence of the scales involved on sea state and first of all on wavelength $\lambda$ and wave steepness $\mu\left(H_{s}\right.$ and $\left.T_{p}\right)$. As shown in this paper (see also B14) the standard 1 second data assimilated in the databases of Ku-band satellite altimetry (e.g. Globwave) are likely relevant to the scaling (8) and thus can be used for estimating wave steepness and wave period. Our experiments with high rate altimetry data $(20 \mathrm{~Hz}$, SGDR - Sensor Geophysical Data Record format) has not shown essential difference in estimates of wave steepness and period corresponding to spatial averaging in a range $3-15 \mathrm{~km}$. Strictly speaking, the averaging of $20 \mathrm{~Hz}$ data in time is not correct because of nonlinearity of dependence of the estimated wave height $H_{s}$ on the altimeter pulse shape. In order to solve this issue proper analysis of individual waveforms (for Envisat RA-2 $1800 \mathrm{~Hz}$ ) is required which is out of the scope of the current study.

The parametric models of sea state from altimetry data operating with one-point measurements [e.g. 12,13] of $H_{s}$ and $\sigma_{0}$ are formally free of the restrictions like those of the physical model B14. But inherent spurious correlation of the measured characteristics bind the performance of a parametric approach when using two-parametric (multi-parametric) dependency. In case of the pair $\left(H_{s}, \sigma_{0}\right)$ the anticorrelation has a physical explanation: higher waves provoke more frequent breaking and, thus, lower reflection from the sea surface (lower $\sigma_{0}$ ). Implicit limitations and drawbacks of parametric approaches will be discussed below for the model by Gommenginger et al. [12, hereinafter G03].

In fact, the parametric models represent the best fit of estimates to in situ buoy measurements. Therefore, these models provide a sort of climatological approximation 'killing' an essential variability of the wave steepness and period. On the contrary instantaneously measured $H_{s}, \nabla H_{s}$ in the physical model B14 preserve the natural variability of estimated parameters.

\section{Two approaches for wave studies in altimetry data}

In this section the features of the parametric and physical approaches are demonstrated for Globwave data [see also 14] via comparison with buoy data and crossovers from different altimeters.

\subsection{Satellite/buoy match-ups. Validity test for the pair $\left(H_{s}, \nabla H_{s}\right)$}

The Globwave database of match-ups accepts buoy and altimeter measurements within the $60 \mathrm{~km}$ and 60 minute span. In order to meet the requirements of the physical constraints stated in sect.2.3, a more rigorous quality control was applied. Match-up distance has been reduced to $30 \mathrm{~km}$ and the time mismatch of buoy and satellite measurements - to 30 minutes. Only the altimetry data with quality flags 'good' in three consecutive points were used for computation of along-track components of the gradient $\nabla H_{s}$. The described constrains have reduced the number of analysed Envisat records to 155 in 2011. 

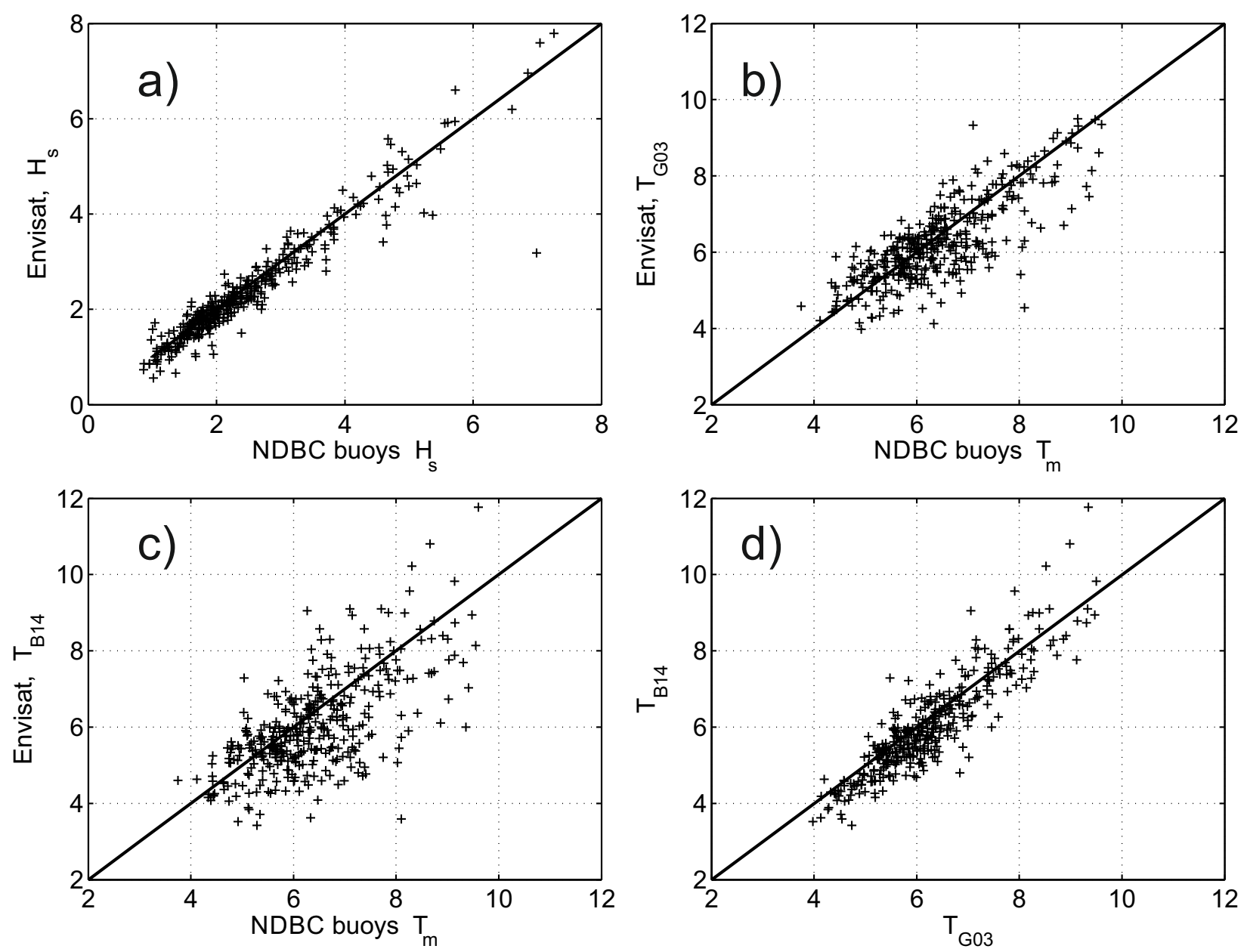

Figure 2. Scatter diagrams for altimeter (Envisat, 2011) and in situ NDBC buoys measurements for the Globwave database of match-ups. a) - significant wave heights (SWH); $b$ ) - wave periods measured by buoys (abscissa) and altimeter (ordinate) estimated by the parametric model G03 [12]; c) - wave periods measured by buoys (abscissa) and altimeter (ordinate) estimated by the physical model B14 [1]; d) - wave periods of G03 vs those of B14 model.

Fig. 2 demonstrates results of the comparison of wave parameters from NDBC and Envisat data. Fig. $2 a$ shows a good agreement for SWH. Mean-over-spectrum wave periods retrieved by the G03 model give an expected correspondence with NDBC measurements (fig.2b). The root-mean-square deviation of the estimates does not exceed 1 second. Similar scatterplot of the wave period with the B14 model (fig.2c) demonstrates a larger dispersion. Nevertheless this dispersion cannot be regarded as the model error only: comparison of wave periods provided by G03 and B14 in fig.2d demonstrates good correspondence of the two models based on essentially different physical principles. Note, that the parametric model G03 (fig.2b) operates with the mean-over-spectrum period $T_{m}$ while the B14 model (2) refers to the spectral peak period $T_{p}$, which in general exceeds $T_{m}$ by $10-20 \%$ [15].

\subsection{Satellite crossovers. Full-gradient and single-track estimates of wave steepness and periods}

Altimeter crossovers are computed for pairs of satellites in order to provide a comprehensive dataset of coincident measurements that can be used to monitor the quality of each sensor and improve their calibration. In the context of the model B14 this data enables to evaluate a full gradient $\nabla H_{s}$ and thus to get a 'full' (not a single-track) estimate of wave steepness (2) and period $T_{p}(1)$. We set the mismatch of satellite measurements to be $r_{c}=5 \mathrm{~km}$ in space and $t_{c}=900 \mathrm{~s}$ in time in order to meet 
physical constraints discussed in sect. 2.3. The assumed characteristic scales of wave field homogeneity and stationarity $r_{c}, t_{c}$ are determined by a simple relationship $r_{c}=t_{c} \times C_{\text {waves }}$ where a characteristic speed $C_{\text {waves }} \approx 5.6 \mathrm{~m} \cdot \mathrm{s}^{-1}$ corresponds to the group velocity of deep water waves with period $T_{p} \approx 7.2$ $\mathrm{s}$ (wavelength $\lambda \approx 75$ meters). This restriction along with quality flags 'good' for three consecutive measurements near a crossover point gives 653 records for the pair Envisat-Jason- 1 for the year 2011.
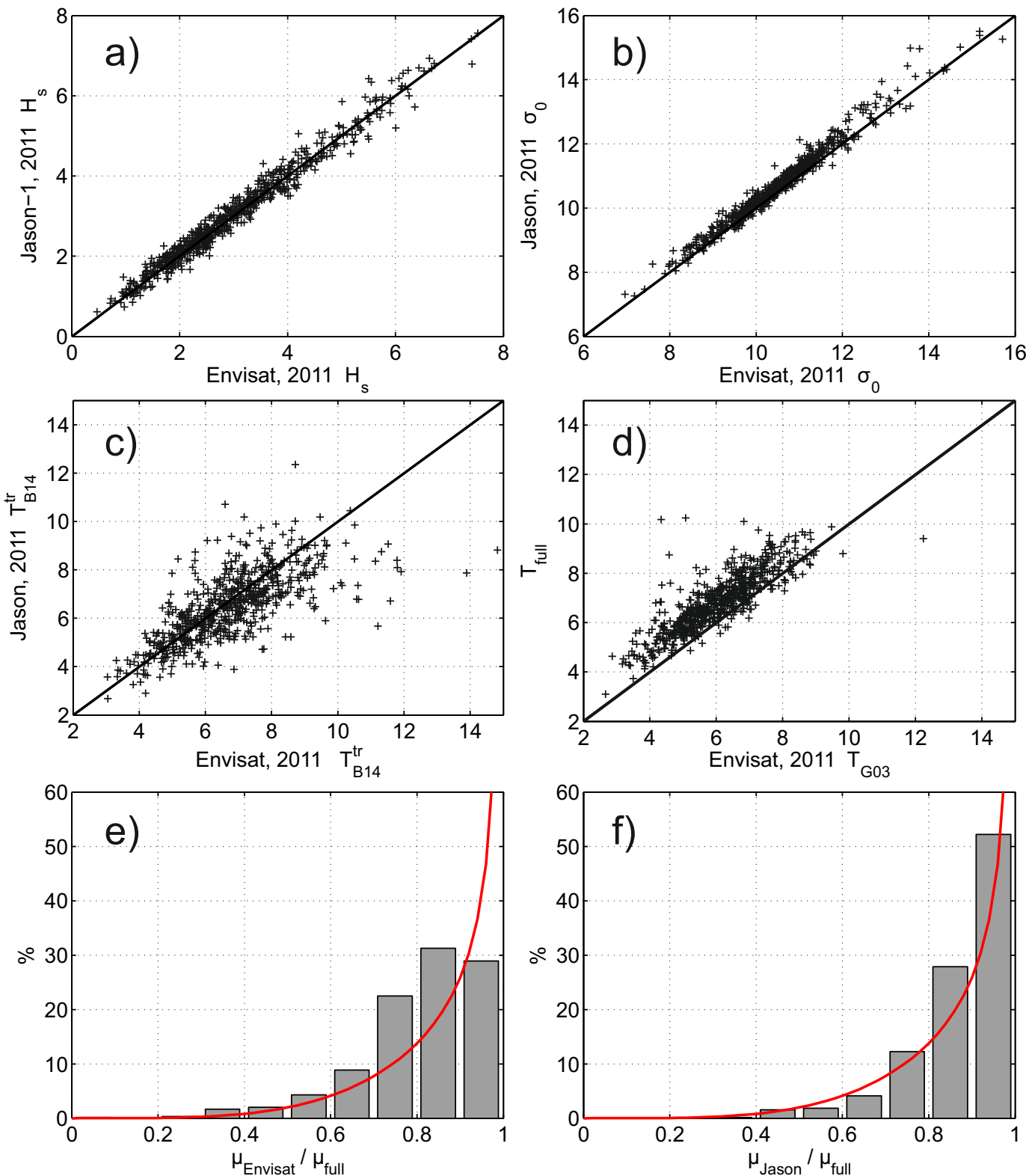

Figure 3. Measurements of Jason-1 and Envisat for the year 2011, totally 653 in cross-over points. Scatter diagrams for $a$ ) - significant wave height; $b$ ) - normalized radar cross-section; $c$ ) - single-track estimates of wave periods by the model B14; $d$ ) - 'full' estimate of wave period $T_{B 14}^{f u l l}$ vs mean-over spectrum wave period $T_{G 03}$ of the model G03 in crossover points; $e, f$ ) - histograms of the ratio $\mu_{t r} / \mu_{f u l l}$ (single-track to the full-gradient estimates) for Envisat (left) and Jason-1 (right) in crossover points. Solid line shows distribution for the probabilistic model (10) of the measured single-track $\mu_{\text {track }}$ with evenly distributed angles between satellite track and wave height spatial gradient $\nabla H_{s}$.

Figs. $3 a, b$ show a good agreement for the conventional pair $\left(H_{s}, \sigma_{0}\right)$ measured with different altimeters. Single-track estimates of wave periods by Envisat and Jason-1 with the model B14 also demonstrate a reasonable correspondence (fig.3c). Full gradient $\nabla H_{s}$ assessment in crossover points 
again gives remarkably good consistence of models of wave period G03 and B14 (fig.2d). The 'full' peak period $T_{p}$ from the B14 model appears to be approximately $15 \%$ higher than the mean wave period of G03 in full agreement with the remarks of the previous paragraph and findings of Babanin and Soloviev [15].

The bottom row presents probability density functions (hereinafter PDF) of the ratio $\mu_{\text {track }} / \mu_{\text {full }}$ of single-track $\mu_{\text {track }}$ and 'full' $\mu_{\text {full }}$ wave steepness for Envisat (fig. $3 e$ ) and Jason-1 (fig.3f). According to (2) the ratio $s=\mu_{\text {track }} / \mu_{\text {full }}$ is proportional to $(\cos \theta)^{1 / 5}\left(\theta\right.$ is angle between gradient $\nabla H_{S}$ and track direction, see right fig.1) and cannot be greater than 1 . We also assume $0 \leq \theta \leq \pi / 2$ because of modulo in (2) and evenness of cosine function. Probability $p_{\theta}(\theta)$ for angle $\theta$ can be easily converted into dependence on this directional factor $s$ :

$$
p_{s}(s)=p_{\theta}(\theta(s))\left|\frac{d \theta}{d s}\right|=p_{\theta}(\theta(s)) \frac{5 s^{4}}{\sqrt{1-s^{10}}}
$$

The uniform (equiprobable) distribution $p_{\theta}(\theta)=2 / \pi\left(\operatorname{PDF}\right.$ is normalized by $\int_{0}^{\pi / 2} p_{\theta}(\theta) d \theta=1$ ) recasted into $p_{s}(s) \equiv p_{s}\left(\mu_{t r} / \mu_{f u l l}\right)$ is shown in figs.3e,f by solid lines. It demonstrates quite close correspondence with histograms for both Jason-1 and Envisat distributions. Theoretical $p_{S}$ (see 9 ) gives probability $85 \%$ of the ratio $\mu_{\text {track }} / \mu_{\text {full }}$ to be 0.75 or higher, i.e. $85 \%$ of single-track measurements underestimate 'full' magnitude of wave steepness $\mu_{\text {full }}$ by less than $25 \%$. An alternative characteristics of quality of single-track measurements of wave steepness can be given in terms of probabilistic mean

$$
\langle s\rangle=\left\langle\frac{\mu_{t r}}{\mu_{f u l l}}\right\rangle=\int_{0}^{1} s p_{s}(s) \frac{5 s^{4} d s}{\sqrt{1-s^{10}}}
$$

For uniform distribution $p_{\theta}=2 / \pi$ one has $\langle s\rangle \approx 0.88$ that makes the single-track estimates acceptable in many cases of interest, say, for global mapping of wave steepness.

The difference between two missions in histograms can be easily explained by domination of zonal circulation over the meridional. As a result, the polar orbit of Envisat (orbit inclination $98.6^{\circ}$ ) exhibits more anisotropic effect of the wind field as compared to Jason-1 (orbit inclination $66^{\circ}$ ).

Thus the single-track estimates provide rather good reference for the 'full' wave steepness and wave period. These estimates can be used as an extension of conventional altimetry of sea state parameters particularly in the context of wave climatology.

\subsection{Gradient measurements for studies of wave dynamics}

As indicated above two models of wave period based on quite different physical principles demonstrate a quantitative agreement. In this regard the new approach with the pair $\left(H_{s}, \nabla H_{s}\right)$ looks an unreasonable complicacy because of computations of spatial gradients $\nabla H_{s}$ that potentially leads to the loss of accuracy. The strongest argument for the novel approach is in additional information provided by along-track variations of the measured parameters. The structured records allow for tracking wave dynamics, thus, extending 'static' wave parameters of point-wise data collections.

Fig.4 presents PDFs of wave parameters with pairs $\left(H_{s}, \sigma_{0}\right)$ (left column) and $\left(H_{s}, \nabla H_{s}\right)$ (right) for three altimetry missions: ERS-2 (1995-2011), Envisat (2002-2012) and Jason-1 (2001-2013) for 2007 when all of them were operating. While all altimeters provide quite close smooth PDFs for $H_{S}$ (fig. $4 b$ ) the PDFs of the normalized radar cross-section $\sigma_{0}$ (fig. $4 a$ ) shown a number of problems. The ERS-2 distribution (red curve) has pronounced peaks which look like artifacts due to inaccurate data processing. The Envisat curve (blue) can be seen as a smoothed approximation of the ERS-2 PDF but it still has twin-peak peculiarity near the distribution extremes. The Jason-1 dramatically differs from the two cases being smoother and with a single maximum shifted to higher $\sigma_{0}$. 



Figure 4. PDFs for $a$ ) - normalized radar cross-section $\sigma_{0} ; b$ ) - significant wave height $H_{s} ; c$ ) - wave period $T_{G 03}$ for the model G03; $d$ ) - wave period $T_{B 14}$ for the model B14; $e$ ) - wave steepness for the model G03; $f$ ) - wave steepness for the model B14; $g$ ) - dimensionless wave height $g H_{s} / U_{\text {alt }}^{2}$ ) vs wave age $g T_{G 03} /\left(2 \pi U_{\text {alt }}\right)$ for the model G03; $\left.h\right)$ - dimensionless wave height $\left.g H_{s} / U_{\text {alt }}^{2}\right)$ vs wave age $g T_{B 14} /\left(2 \pi U_{a l t}\right)$ for the model B14.

Calibration/validation procedures (individual for each altimeter) does not reveal this evident shortcoming of retrieval $\sigma_{0}$ from altimeter pulses when being used for parameterization of wave periods and near-surface wind. In fig. $4 c$ the distribution of wave periods estimated with the $\sigma_{0}$-based G03 model looks quite regular: pronounced oscillations of the $\sigma_{0}$ PDF in fig. $4 a$ disappear. Possible 
explanation of this effect can be found in a strong correlation of $H_{s}$ and $\sigma_{0}$ : their combination $\sigma^{0} H_{s}^{2}$ in the resulting expression for wave period [eq. 4 in $12, \sigma^{0}$ - the normalized radar cross-section in the authentic non- $\mathrm{dB}$ form] compensates the oscillations. While these peaks disappear in PDF of wave period they still remain visible for wave steepness of ERS-2 in fig.4e.

The pair $\left(H_{s}, \nabla H_{s}\right)$ provides smooth patterns for estimates of wave periods (fig. $4 d$ ) with a lag of the ERS-2 curve and, what is more important, with essentially wider distributions in comparison with the G03 estimates (fig.4c). The distribution for wave steepness in fig. $4 f$ reveals another issue which is a high noise of wave height gradient. It makes estimates of wave steepness from the ERS-2 data useless while those of wave period remain acceptable because of lower exponent $1 / 10$ in (1).

Comparison of the wave steepness distributions shows qualitative disagreement of the conventional pair $\left(H_{s}, \sigma_{0}\right)$ and the new one $\left(H_{s}, \nabla H_{s}\right)$ (figs. $\left.4 e, f\right)$. In both cases distributions are localized in approximately the same range $0.05<\mu<0.1$ but the 'conventional' estimate (fig.4e) gives sharper patterns (note different scales of ordinates in the panels). Similar sharpening of PDF for the G03 model can be seen for wave periods (cf. fig.4c,d). This qualitative dissimilarity of probabilistic features of B14 and G03 (and more generally disagreement between physical and parametric models) reflects conceptual difference of the pairs of measured quantities $\left(H_{s}, \nabla H_{s}\right)$ or $\left(H_{s}, \sigma_{0}\right)$. As noted above (see sect.2.3) the physical model B14 directly assesses instantaneous values based on explicit physical assumptions while its parametric counterpart G03 is looking for the best fit to reference data (e.g. ocean buoys) of an inherently probabilistic dependence on $\left(H_{s}, \sigma_{0}\right)$. In other words, a parametric approach targets to the most probable estimates rather than the instantaneous values.

Strong argument for improved relevance of the B14 approach can be found in distributions of dimensionless wave height and period scaled by wind speed. Figs. $4 g, h$ demonstrate dramatic discrepancy of two approaches in their ability to reflect sea wave dynamics. PDF is plotted for dimensionless wave period $\tilde{T}=g T_{p} /\left(2 \pi U_{\text {alt }}\right)$ (wave age) and dimensionless wave height $\tilde{H}=$ $g H_{s} / U_{a l t}^{2}$. The similar approach based on physical scale of wind speed [16] is widely used in sea wave studies [e.g. 10,17]. It provides a ground for quantitative predictions like the Toba [18] 3/2 law, 5/3 law of Hasselmann et al. [19], 4/3 power-law dependence of Zakharov and Zaslavsky [20] or one of fully developed sea [21]. These models demonstrate both an effectiveness and a deficiency of the wind speed scaling: a large scatter of experimental data is observed using dimensionless $\tilde{T}$ and $\tilde{H}$ [e.g. 22-24]. The scatter reflects a non-universality of wind-wave coupling when features of wind over waves (gustiness, stratification etc.) affect the wave growth.

The existing non-universality or in other words diversity of wave dynamics in terms of dimensionless $\tilde{T}$ and $\tilde{H}$ is seen when following the B14 approach (fig.4h). Power-law dependencies corresponding to the Toba [18] 3/2 law (solid line) and to constant values of wave steepness $\mu=0.1$ and $\mu=0.04$ (dashed) are given for reference in fig. $4 h$. The distribution pattern covers both cases of rough sea $(\mu \approx 0.1)$ and of the mature wind sea of Pierson and Moskowitz [21] ( $\mu \approx 0.04)$. The shrinking of the G03 distribution can be treated as a corruption of wave physics that implies rather wide dispersion of the PDF due to diversity of effects of wave growth. The derivation itself of the parametric model G03 [see sect.3 in 12] postulates the proximity to a constant wave steepness. This is not consistent with the dynamical laws predicting a decrease of wave steepness with growing wave age. Alternatively, a remarkable spreading of the B14 PDF to high wave ages matching the line of the Toba law can be treated as a manifestation of the effect of nonlinear transfer to low frequencies [25].

\subsection{Global distributions of wave steepness: a snapshot}

High quality of altimetry measurements of wave height $H_{s}$ and near-surface wind speed $U_{\text {alt }}$ has been demonstrated in many studies [e.g. 26,27]. Recently good agreement has been found between global distributions of wave periods retrieved from altimetry data and Voluntary Observing Ship data (VOS) $[14,28]$. It provides us with a ground for incorporating the altimetry data into conventional climatology of sea waves [e.g. 7]. In general this climatology operates with mean values averaged both in space (in coordinate boxes) and time (monthly, annually). The geographical distributions of 
mean wave heights and periods appear quite representative and useful for further analysis in the context of climate changes [7,29] and wave extremes [30].Similar global mapping of wave steepness looks questionable because the latter varies in an incomparably narrower range than wave heights or periods.

Fig. 5 shows a geographical distribution of wave steepness in 2011 averaged over boxes $2^{\circ} \times 2^{\circ}$ for Envisat data. Wave steepness has been estimated with the model B14 (eq.2 and top of fig.5) and the parametric model G03 (bottom fig.5) with $\mu$ defined by (3). Both maps show similar general patterns with pronounced latitudinal dependence and maxima in the Southern and Northern Atlantic and Pacific Ocean. Along with systematic underestimation of wave steepness by G03 we can also see descrepancies in particular enclosed basins (Mediterranean, Mexican Gulf etc.) and marginal seas (e.g. Indonesian seas).

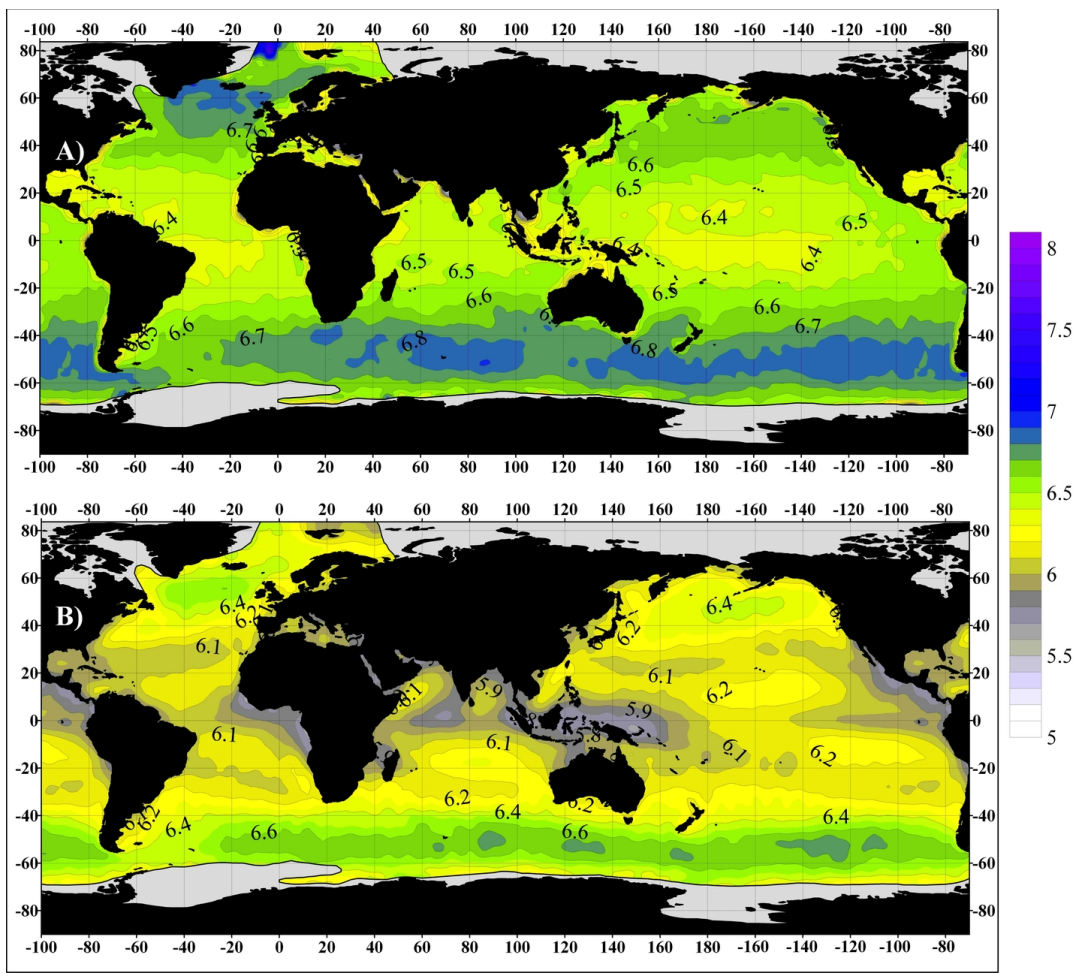

Figure 5. Global distribution of wave steepness derived from Envisat data in 2011. Top - physical model by Badulin [1]; bottom - parametric approach by Gommenginger et al. [12]

Fig. 5 provides a very preliminary look at wave steepness climatology. Relatively low variability (within few percents of typical magnitude) does not reflect a crucial role of this physical parameter in wave dynamics. In fact such key wave parameters as energy and momentum fluxes are heavily nonlinear functions of wave steepness.

Fig.6 demonstrates the key message of the above sections on the effect of specific approach (parametric or physical) on climatological constructions. Probability density functions are presented for the authentic data set (Envisat, 2011) of wave steepness (left panel) and for values averaged over $2^{\circ} \times 2^{\circ}$ coordinate boxes (right panel). On the left panel of fig. 6 the physical model B14 (grey) gives a rather wide distribution of the authentic data (as expected from figs. $4 f, h$ ) while the parametric approach G03 contracts the values in a narrow range $0.05-0.08$ (black). The physical approach at least doubles this range and thereby reflects an essentially wider variability of the instantaneous wave steepness. The parametric model G03 in turn exhibits relatively weak dispersion of the most probable wave steepness. 
The distributions of mean values of wave steepness, averaged over coordinate boxes $2^{\circ} \times 2^{\circ}$ on the right panel of fig.6, look remarkably different for the models G03 and B14 (cf. left and right figs. 6). The dispersion of the first one (G03) remains roughly the same for authentic data while the width of the sampling B14 distribution squeezes by a factor $\sqrt{N_{2 \times 2}}\left(N_{2 \times 2}\right.$ being the number of data in $2^{\circ} \times 2^{\circ}$ coordinate box, $N_{2 \times 2} \approx 100-400$ in our example). Such squeezing of the mean value distribution indicates that the sets of the steepness, evaluated within B14 in each $2^{\circ} \times 2^{\circ}$ box, are the realizations of essentially the same random process. However, the best-fit model G03 does not reveal such universality.

This result suggests quite surprising climatological interpretation:

climatology of wave steepness is quite indifferent to the geographical region in contrast to wave height $H_{s}$ and period $T_{p}$ that demonstrate significant regional variations.

In this way an incorporating wave steepness into today 'classic' wave climatology requires more subtle methods of data analysis. The found universality of wave steepness climate within the B14 model does not mean a universality of wave dynamics in space and time. A thorough analysis of the global wave steepness distributions with two fundamentally different methods allows us to broaden our understanding of the wave dynamics.
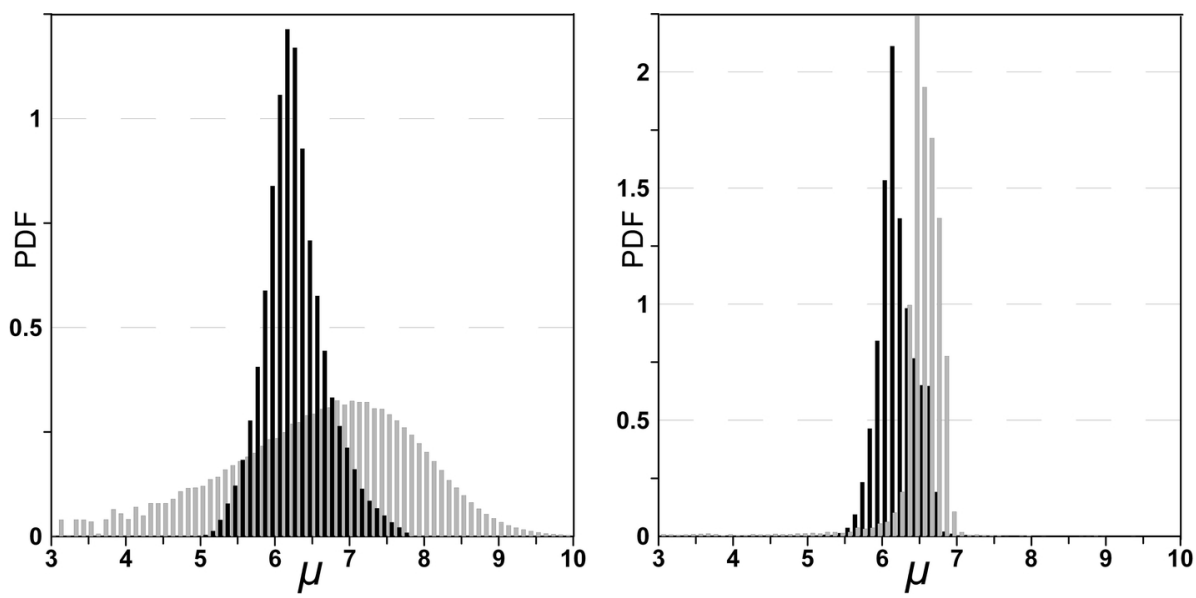

Figure 6. Probability density functions (PDF) for wave steepness estimates made within the parametric model by Gommenginger et al. [12, black, eq.3] and the physical one by Badulin [1, grey, eq.2]. Left - PDF for individual estimates of wave steepness; Right - PDF for the set of $2^{\circ} \times 2^{\circ}$ averaged wave steepness.

\section{Concluding remarks}

Satellite altimetry is a valuable source of data for ocean studies. The applications of this data are broad-ranging though measurements of sea surface elevation remain the primary goal of the today altimetry. These measurements provide an input for assessments of large-scale dynamics of the ocean where sea level variations are considered as a driver of ocean currents together with the Coriolis force. In this way the field of the variations, i.e. spatial gradients of sea level are analyzed and processed.

Recent progress in remote sensing technologies allows measurements of sea surface roughness (wave height) and hence its along-track variations. Potentially these records contain information on along-track wave dynamics but altimetry measurements of sea waves continue to be treated as point-wise, static data. Wave period and near surface wind speed are retrieved from the data with statistical methods. However all the parametric models lead us to the most probable values for a given combination of directly measured wave height $H_{s}$ and normalized radar cross-section $\sigma_{0}$. Thus valuable information of the evolution of wave field along the satellite track is not used. The idea to convert along-track records of the directly measured wave height $H_{s}$ into unavailable parameters 
of wind-sea coupling looks promising. The above mentioned estimates of large-scale currents by altimetry methods provide an encouraging example and useful physical analogy.

This analogy is not straightforward as we discuss this issue in sect.2.1. Dynamical method for large-scale currents is based on reduction of primitive equations of geophysical hydrodynamics when a number of terms are assumed to be small. Negligibility of these terms can be formulated explicitly (e.g. as a smallness of the Rossby number). It is not the case of the model B14 (see sect. 2.3). Firstly, this model is based on a number of assumptions and hypotheses of statistical theory of wind-driven seas: evolution of the wave field should be slow enough on scales of wave periods (wave lengths). Secondly, nonlinear transfer should be a dominating term in the Hasselmann equation for simple linking of total (integral) wave input and instantaneous sea state [5,9]. Finally, altimetry measurements (the footprint and intervals between consecutive soundings) should provide observability of the above physical link.

The existence of the physical link and subsequent converting of along-track records into sea state parameters implies instantaneous values in contrast to the most probable assessment by parametric models. This is an important point that shows promising prospects for extending our analysis of the altimetry data. The model B14 does not pretend to check or replace the previously proposed methods but does provide additional information. The combination of the parametric (e.g. G03) and physical (B14) models make a ground for advanced wave climatology where mean (the most probable) parameters of the sea state can be extended by estimates of their variations.

The key dimensionless parameter of wave dynamics - wave steepness is used extensively in this work. First, it appears as a result of remarkably simple conversion (2) of the measured along-track variations of wave height into essential information on instantaneous sea state (wave steepness and period). Secondly, we propose to extend the conventional wave climatology by this parameter. Global spatial distributions of this value are presented for the first time, in authors' knowledge. These distributions discover an intriguing feature of wave steepness: its geographical climatic variability is remarkably low in contrast to conventional wave parameters (wave height and period) that show pronounced regional (first of all, latitudinal) dependence. This universality looks promising though it requires further thorough analysis.

Simulations of wave field evolution with state-of-the-art third generation spectral models (WaveWatch III, WAM etc.) can be used in order to verify results presented above. These models demonstrate reasonable performance in reproducing sea state across the globe. At the same time, the model application at scales of the altimetry measurements when spatial and temporal scaling should follow strict physical constraints (see sect.2.3), requires additional tuning of the model configurations. Verification of the results of this work within the spectral wave models is a necessary further steps.

We summarize the paper by brief discussion of its key points.

1. New physical model of wave steepness is designed in order to expand the applications of altimetry data. The model implements the method previously used for the assessment of large-scale currents from altimetry based on geostrophic balance. Both of these approaches are based on the use of spatial gradients of measurable sea state parameters as an extension or/and an alternative to conventional point-wise characteristics $H_{s}, \sigma_{0}$ of satellite altimetry. The gradient of significant wave height $H_{s}$ can only be partially estimated as an along-track derivative and thus the routine along-track altimetry measurements tend to underestimate wave steepness values. The essentially nonlinear dependence (2) makes these estimates acceptable for wave studies. The results are supported by data analysis of buoy-altimeter match-ups and crossover points of altimeters that demonstrates the relevance of the physical model B14 itself and the applicability of the single-track estimates;

2. The proposed physical model does not contain any empirical parameters and therefore does not necessarily need to go through calibration. At the same time the model requires a correspondence on a number of physical scales. The analysis of standard 1-second altimetry data reveals reasonable agreement between these scales and those of the today Ku-band altimeters. It is important to point out that this does not guarantee the general applicability of this method, for 
example, for prospective Ka-band altimeters (e.g. SARAL/AltiKa) due to possible issues with fitting the scaling (6). We consider a good agreement with the standard Ku-band altimetry data with a certain caution and look forward to analyze the implication of this method to different physical scales and new altimetry data;

3. The transparent physics of the new approach ensure operations with instantaneous values of the estimated sea state parameters (wave steepness and period). This approach is conceptually different from widely-used parametric approaches that operate with the best-fit approximation dependencies and thus provide the most probable estimates for the point-wise measurements of $H_{s}$ and $\sigma_{0}$. We considered one of these models - G03 [12] as a representative example in order to analyze and specify the discrepancy of two approaches. According to our analysis the suggested methods demonstrate a good agreement for estimated wave steepness and periods PDFs for different satellite missions. At the same time the physical model B14 shows wide distributions of their instantaneous estimates while the parametric G03 clenches them up. The sharpening of the G03 PDFs for $T_{p}$ and $\mu$ as compared to those for B14 (see fig.4) reflects the probabilistic nature of the parametric approach. The G03 model (as well as other parametric models) looks for the best fit in a sub-space of just two measured parameters in, very likely, wider space of physical arguments that affect wave evolution. For two-dimensional distributions in dimensionless wave periods $\tilde{T}$ and heights $\tilde{H}$ (see fig. $4 g, h$ ) the squeezing can be interpreted as a corruption of inherent features of wind-wave coupling when the wave steepness appears to be close to a constant value;

4. Global spatial distribution of wave steepness for both approaches B14 and G03 has been presented for the first time. This provides a motivation for further discussion on potential incorporation of wave steepness into existing sea wave climatologies. In contrast to other wave parameters a trivial averaging of the wave steepness was shown to conceal essential features of the presented physical approach. More subtle procedures are therefore required to develop the proposed method;

5. The validity of the physical model of wave steepness (2) remains a subject of thorough studies. Along with the issue of validity of the asymptotic model (the so-called split-balance model of wind-driven seas [see 5,31] our approach implies an additional assumption of quasi-stationarity of the wave field. The time derivative $\partial H_{s} / \partial t$ should be much less than the convective term $C_{g} \nabla H_{S}$ ( $C_{g}$ is wave group velocity) that allows to reduce the problem to a fetch-limited setup. All the model assertions would be validated against the spatial estimates of wave period $T_{p}$ available from global wave model products such as WAM or Wavewatch. These models show quite reasonable performance in reproducing wave periods across the globe. At the same time, their application to the altimetry measurements requires special solutions when spatial and temporal scaling should follow strict physical constraints of sect.2.3. It makes such verification of our results to be an important point of the authors' agenda.

Acknowledgments: Open access data of the ESA initiative Globwave (http://globwave.ifremer.fr/) and portal AVISO (http:/ / www.aviso.altimetry.fr/en/home.html) have been used in this work. The results of parts 1,2 were obtained in the framework of the state assignment of FASO Russia (themes No. 0149-2018-0017, 0149-2018-0023). Database of the altimetry data has been compiled within the grant of Russian Foundation for Basic Research No. 14-05-00479. Data analysis has been supported by Russian Science Foundation (No. 14-50-00095). Authors are thankful for the continuing support of these agencies. Authors also appreciate valuable criticism of the first version of the work by Prof. Luigi Cavaleri.

Author Contributions: S.I. Badulin developed the theoretical model of the sea wave steepness from altimetry data. V.G. Grigorieva contributed to the data analysis. A.V. Gavrikov and M.V. Krinitskiy performed processing of alimetry data. V.V Geogjaev adopted the Globwave database of the buoy/satellite match-ups and satellite crossovers for the goals of this study. M.Yu. Markina estimated the theoretical model validity within the spectral wave model WaveWatch III.

Conflicts of Interest: The authors declare no conflict of interest. The founding sponsors had no role in the design of the study; in the collection, analyses, or interpretation of data; in the writing of the manuscript, and in the decision to publish the results. 


\section{References}

1. Badulin, S.I. A physical model of sea wave period from altimeter data. J. Geophys. Res. Oceans 2014, 119.

2. Brown, G. The average impulse response of a rough surface and its applications. IEEE Trans. Antennas Propagat. 1977, 25, 67-74.

3. Barrick, D.; Lipa, B. Analysis and interpretation of altimeter sea echo. Advances in Geophysics 1985, $27,60-100$.

4. Zakharov, V.E.; Lvov, V.S.; Falkovich, G. Kolmogorov spectra of turbulence. Part I; Springer, Berlin, 1992.

5. Badulin, S.I.; Babanin, A.V.; Resio, D.; Zakharov, V. Weakly turbulent laws of wind-wave growth. J. Fluid Mech. 2007, 591, 339-378.

6. Badulin, S.I.; Zakharov, V.E. Ocean swell within the kinetic equation for water waves. Nonl. Proc. Geophys. 2017, 24, 237-253.

7. Gulev, S.; Grigorieva, V.; Sterl, A.; Woolf, D. Assessment of the reliability of wave observations from voluntary observing ships: Insights from the validation of a global wind wave climatology based on voluntary observing ship data. J. Geophys. Res. 2003, 108, 3236.

8. Hasselmann, K. On the nonlinear energy transfer in a gravity wave spectrum. Part 1 . General theory. J. Fluid Mech. 1962, 12, 481-500.

9. Zakharov, V.E.; Badulin, S.I. On Energy Balance in Wind-Driven Seas. Doklady Earth Sciences 2011, $440,1440-1444$.

10. Cavaleri, L.; Alves, J.H.G.M.; Ardhuin, F.; Babanin, A.; Banner, M.; Belibassakis, K.; Benoit, M.; Donelan, M.; Groeneweg, J.; Herbers, T.H.C.; Hwang, P.; Janssen, P.A.E.M.; Janssen, T.; Lavrenov, I.V.; Magne, R.; Monbaliu, J.; Onorato, M.; Polnikov, V.; Resio, D.; Rogers, W.E.; Sheremet, A.; Smith, J.M.; Tolman, H.L.; van Vledder, G.; Wolf, J.; Young, I. Wave modelling - the state of the art. Progr. Ocean. 2007, 75.

11. Zakharov, V.E. Energy balance in a wind-driven sea. Phys. Scr. 2010, T142, 014052.

12. Gommenginger, C.P.; Srokosz, M.A.; Challenor, P.G.; Cotton, P.D. Measuring ocean wave period with satellite altimeters: A simple empirical model. Geophys. Res. Lett. 2003, 30, 2150.

13. Mackay, E.B.L.; Retzler, C.H.; Challenor, P.G.; Gommenginger, C.P. A parametric model for ocean wave period from Ku-band altimeter data. J. Geophys. Res. 2008, 113.

14. Gavrikov, A.V.; Krinitsky, M.A.; Grigorieva, V.G. Modification of Satellite Altimetry Database GlobWave for Diagnosis of Sea Wave Fields. Oceanology 2016, 56, 301-306.

15. Babanin, A.N.; Soloviev, Y.P. Field investigation of transformation of the wind wave frequency spectrum with fetch and the stage of development. J. Phys. Oceanogr. 1998, 28, 563-576.

16. Kitaigorodskii, S.A. Applications of the theory of similarity to the analysis of wind-generated wave motion as a stochastic process. Bull. Acad. Sci. USSR, Geophys. Ser., Engl. Transl. 1962, N1, 105-117.

17. Hwang, P.A.; Teague, W.J.; Jacobs, G.A.; Wang, D.W. A statistical comparison of wind speed, wave height and wave period derived from satellite altimeters and ocean buoys in the Gulf of Mexico region. J. Geophys. Res. 1998, 103, 10451-10468.

18. Toba, Y. Local balance in the air-sea boundary processes. Part I. On the growth process of wind waves. J. Oceanogr. Soc. Japan 1972, 28, 109-121.

19. Hasselmann, K.; Ross, D.B.; Müller, P.; Sell, W. A parametric wave prediction model. J. Phys. Oceanogr. 1976, 6, 200-228.

20. Zakharov, V.E.; Zaslavsky, M.M. Dependence of wave parameters on the wind velocity, duration of its action and fetch in the weak-turbulence theory of water waves. Izv. Atmos. Ocean. Phys. 1983, 19, 300-306.

21. Pierson, W.J.; Moskowitz, L.A. A proposed spectral form for fully developed wind seas based on the similarity theory of S. A. Kitaigorodsii. J. Geophys. Res. 1964, 69, 5181-5190.

22. Donelan, M.; Skafel, M.; Graber, H.; Liu, P.; Schwab, D.; Venkatesh, S. On the growth rate of wind-generated waves. Atmosphere Ocean 1992, 30, 457-478.

23. Abdalla, S.; Cavaleri, L. Effects of wind variability and variable air density on wave modeling. J. Geophys. Res. 2002, 107, doi:10.1029/2000JC000639.

24. Hwang, P.A.; Wang, D.W. Field measurements of duration-limited growth of wind-generated ocean surface waves at young stage of development. J. Phys. Oceanogr. 2004, 34, 2316-2326.

25. Glazman, R. Effects of sea maturity on satellite altimeter measurements. J. Geophys. Res. 1990, 95, $2857-2870$. 
491

492

493

494

495

26. Young, I.R.; Zieger, S.; Babanin, A.V. Global Trends in Wind Speed and Wave Height. Science 2011, 332, 451-455.

27. Young, I.; Sanina, E.; Babanin, A. Calibration and cross-validation of a global wind and wave database of Altimeter, Radiometer and Scatterometer measurements. J. Atmos. Ocean. Technol. 2017.

28. Grigorieva, V.G.; Badulin, S.I. Wind Wave Characteristics Based on Visual Observations and Satellite Altimetry. Oceanology 2016, 56, 19-24.

29. Gulev, S.K.; Grigorieva, V.G. Variability of the winter wind waves and swell in the North Atlantic and North Pacific as revealed by the voluntary observing ship data. Journal of Climate 2006, 19, 5667-5685.

30. Grigorieva, V.; Gulev, S. Extreme waves in visual wave observations by VOS. Proceedings of the Rogue Waves 2008 Workshop, October 13-15, Brest; Olagnon, M.; Prevosto, M., Eds., 2008, pp. 41-49.

31. Badulin, S.I.; Pushkarev, A.N.; Resio, D.; Zakharov, V.E. Self-similarity of wind-driven seas. Nonl. Proc. Geophys. 2005, 12, 891-946. 